\title{
Recursos pessoais e contextuais preditores de perceção de saúde na adolescência
}

\section{Personal and contextual developmental assets predictors of health perception in adolescence}

\section{Recursos personales y contextuales predictores de percepción de salud en la adolescencia}

\author{
Ana Soares ${ }^{1}$ ORCID 0000-0001-8304-0785 \\ José L. Pais-Ribeiro ${ }^{2}$ ORCID 0000-0003-2882-8056 \\ Isabel Silva ${ }^{3}$ ORCID 0000-0002-6259-2182 \\ ${ }^{12}$ Faculdade de Psicologia e de Ciências da Educação, Universidade do Porto. Portugal \\ ${ }^{3}$ Universidade Fernando Pessoa, Porto. Portugal
}

\begin{abstract}
Resumo: A Perceção de Saúde é o produto da interação entre características pessoais e condições contextuais. Esta reflete os recursos da pessoa, assim como consiste numa avaliação dinâmica, um julgamento que reflete estado e processo de desenvolvimento. O Modelo de Recursos do Desenvolvimento - Developmental Assets ${ }^{\circledR}$ - proporciona uma abordagem holística na compreensão do desenvolvimento, foco em Recursos que podem ser explorados quer ao nível individual, quer contextual de modo a propiciar aos adolescentes um desenvolvimento saudável. O objetivo do presente estudo consiste em explorar a relação entre a Perceção de Saúde e a experiência de Recursos do Desenvolvimento. Foi avaliada uma amostra de conveniência constituída por 503 estudantes portugueses, com idades compreendidas entre os 13 e 19 anos $(M=16 ; D P=1,2)$, maioritariamente do género feminino (63\%). Os resultados indicam que melhor Perceção de Saúde está associada a maior experiência de Recursos do Desenvolvimento. Os dados sugerem o impacto de um subconjunto de Recursos na predição de Saúde, constituindo os Recursos Internos os preditores mais fortes. Sugerese a utilidade teórica e prática da utilização do Modelo de Recursos do Desenvolvimento para a compreensão da Perceção de Saúde em adolescentes.
\end{abstract}

Palavras-chave: Perceção de Saúde; Recursos do Desenvolvimento; Perspetiva do Desenvolvimento Positivo na Adolescência; Desenvolvimento Saudável

Abstract: Self-Rated Health is the product of the interaction between personal characteristics and contextual conditions. It reflects the resources of the person, and it consists in a dynamic evaluation, i.e., a personal judgment that reflects both a point-in-time status and a developmental process. The Developmental Assets ${ }^{\circledR}$ framework provides a holistic approach in to the understanding of development, which focuses on resources that can be explored both at individual and contextual levels, in order to foster a healthy development. The purpose of this research is to explore the relationship between adolescents' Perception of Health and their experience of Developmental Assets. A convenience sample of 503 Portuguese students was evaluated, mean age of 16 years $(S D=1.2), 63 \%$ of female gender. Results indicate that better Health Perception is associated with greater experience of Developmental Assets. Results suggest the impact of a subset of Developmental Assets on Health prediction, with Internal Assets being the strongest predictors. Results suggest the theoretical and practical utility of the Developmental Assets ${ }^{\circledR}$ framework for the understanding of Health Perception in adolescents.

Keywords: Self-Rated Health; Developmental Assets; Positive Youth Development; Healthy Development 
Resumen: La Percepción de Salud es el producto de la interacción entre características personales y condiciones contextuales. Esto refleja los recursos de la persona, así como consiste en una evaluación dinámica, un juicio que refleja el estado y el proceso de desarrollo. El Modelo de Recursos del Desarrollo - Developmental Assets ${ }^{\circledR}$ - proporciona un enfoque holístico en la comprensión del desarrollo, foco en Recursos que pueden ser explorados tanto a nivel individual, como contextual para propiciar a los adolescentes un desarrollo saludable. El objetivo del presente estudio consiste en explorar la relación entre la Percepción de Salud y la experiencia de Recursos del Desarrollo. Se evaluó una muestra de conveniencia constituida por 503 estudiantes portugueses, con edades comprendidas entre los 13 y 19 años $(M=16, D E$ $=1.2$ ), mayoritariamente del género femenino $(63 \%)$. Los resultados indican que la mejor percepción de la salud está asociada a la mayor experiencia de recursos del desarrollo. Los datos sugieren el impacto de un subconjunto de Recursos en la predicción de Salud, constituyendo los Recursos Internos los predictores más fuertes. Se sugiere la utilidad teórica y práctica de la utilización del Modelo de Recursos del Desarrollo para la comprensión de la Percepción de Salud en adolescentes.

Palabras clave: Percepción de Salud; Recursos del Desarrollo; Perspectiva del Desarrollo Positivo en la Adolescencia; Desarrollo Saludable

Recebido: 12/02/2019

Aceito: $16 / 08 / 2020$

Como citar:

Soares, A., Pais-Ribeiro, J. L., \& Silva, I. (2020). Recursos pessoais e contextuais preditores de perceção de saúde na adolescência. Ciencias Psicológicas, 14(2), e-2263. doi: https://doi.org/10.22235/cp.v14i2.2263

Correspondência: Ana Soares, Faculdade de Psicologia e de Ciências da Educação da Universidade do Porto. Correspondencia: Avenida Joaquim Ferreira Araújo, no 11, 4730-280, Marrancos, Vila Verde. E-mail: anasofiabsoares@gmail.com. José L. Pais-Ribeiro, Faculdade de Psicologia e de Ciências da Educação da Universidade do Porto. E-mail: jlpr@fpce.up.pt. Isabel Silva, Universidade Fernando Pessoa, Porto. E-mail: isabels@ufp.edu.pt

Em geral, como classificaria a sua Saúde? Fraca, razoável, boa, muito boa ou excelente? Esta simples questão frequentemente designada de Saúde Percebida - é também comummente referida como, Perceção de Saúde, Saúde Subjetiva, Autoavaliação da Saúde (Benyamini, 2011a, 2011b), sendo utilizada em inúmeros estudos. Ela é, por exemplo, utilizada no Eurostat para avaliar a saúde dos europeus (Eurostat, 2020).

Perceções subjetivas das pessoas sobre a sua saúde captam fatores físicos, psicológicos e sociais, em congruência com a visão holística da saúde compreendida no modelo biopsicossocial, assim como com a definição de saúde da Worl Health Organization (1948) enquanto um estado de completo bem-estar físico, mental, e social, e não meramente a ausência de doença ou enfermidade (Benyamini, 2008, 2011a; Pais-Ribeiro, 2005). Em adolescentes, observa-se que perceções subjetivas da saúde captam esta visão holística da saúde (Joffer, Jerdén, Öhman, \& Flacking, 2016). A investigação indica que perceções subjetivas da saúde global sugerem ir mais além de um modelo médico de saúde como ausência de doença, na medida em que abrangem todo o continuum doença bem-estar (Bishop \& Yardley, 2010), bem como proporcionam sumários compreensivos do conjunto de fatores que as pessoas consideram como parte constituinte do seu próprio conceito de saúde (Benyamini, 2008; 2011a; Pais-Ribeiro, 2005).

A investigação demonstrou que a Perceção de Saúde está associada a doenças e sintomas, capacidade funcional, utilização de serviços de saúde, utilização de medicação, saúde mental, 
suporte social e fatores socioeconómicos, sugerindo que estes são fortemente afetados pelo afeto positivo e não apenas pela doença, incapacidade ou afeto negativo (Benyamini, 2008; Benyamini, Idler, Leventhal, \& Leventhal, 2000; Pais-Ribeiro, 2005; Pinquart, 2001). A investigação indica ainda que a perceção subjetiva de saúde não só se encontra relacionada com várias medidas de saúde, mas também é melhor preditor de longevidade do que os registos médicos ou as descrições de doenças (Benyamini \& Idler, 1999; Doiron, Fiebig, Johar, \& Suziedelyte, 2015; Idler \& Benyamini,1997; Jylhä, 2009). Tais resultados na investigação indicam a validade de perceções subjetivas de saúde, levando a que estas sejam atualmente consideradas como um indicador para medir a saúde, em vez de medidas mais detalhadas de saúde (Benyamini, 2008). Em suma, nas palavras de Benyamini (2011a, p. 305), considerando-se perceções de saúde, "o subjetivo é o objetivo".

Benyamini (2011b, p.1410) salienta que a validade da Perceção de Saúde como um preditor de resultados de saúde futuros reside em quatro explicações 1) esta é mais inclusiva do que as covariáveis utilizadas em vários estudos; 2) consiste numa avaliação dinâmica, que reflete o julgamento relativo à trajetória da saúde e não apenas a saúde atual, isto é, num ponto definido do tempo - também designada "hipótese de trajetória"; 3) influencia comportamentos que subsequentemente afetam o estado de saúde; e 4) reflete os recursos da pessoa que refletem ou até mesmo afetam a capacidade de lidar com ameaças à saúde.

Deste modo, salienta-se que a investigação indica que a Perceção de Saúde reflete os recursos da pessoa e está relacionada tanto a recursos externos, como por exemplo, suporte social, assim como a recursos internos, como por exemplo, otimismo, controlo percebido (Benyamini, 2011a, 2011b; Benyamini, Blumstein, Murad, \& Lerner-Geva, 2011). A inclusão destes recursos, por sua vez, diminui frequentemente a contribuição da Perceção de Saúde na predição da saúde futura, no entanto, ainda carece de explicação se tal se deve pelo facto de estes recursos, especialmente os internos, refletirem o estado de saúde, ou porque estes têm um efeito causal na saúde futura (Benyamini, 2011b). Benyamini (2011b) explica que os recursos da pessoa, externos e internos, constituem fatores que podem levar a diferentes trajetórias de saúde futura.

As perceções de saúde e doença estão inseridas no contexto pessoal e cultural, pelo que fazem parte do processo de socialização, sendo, portanto, o produto de uma interação entre características pessoais, as ações intencionais de agentes socias como família, pares e sistema educacional, assim como da exposição a várias condições do contexto social no qual a pessoa está inserida (Benjamin, 2011a). Neste sentido, surge no âmbito da Perspetiva do Desenvolvimento Positivo na Adolescência, o conceito de Recursos do Desenvolvimento - "Developmental Assets" ${ }^{\circledR}$ - desenvolvido pelo Search Institute (Benson, 2006), com o objetivo de descrever o que os jovens necessitam para um desenvolvimento bem-sucedido (Benson, Scales, \& Syvertsen, 2011). O Modelo associa características, competências, capacidades e qualidades pessoais (Recursos Internos) a características ecológicas positivas (Recursos Externos) assumindo que estas se encontram dinamicamente interligados (Benson et al., 2006), que, em combinação, para além de prevenirem comportamentos comprometedores de resultados de saúde (Benson \& Scales, 2009), propiciam um desenvolvimento otimamente bem-sucedido na adolescência (Benson et al., 2006; Scales, Benson, Leffert, \& Blyth, 2000; Scales et al., 2006). O Modelo de Recursos de Desenvolvimento é apresentado de forma sumária na Tabela 1. 
Tabela 1

Modelo de Recursos do Desenvolvimento - Developmental Assets ${ }^{\circledR}$

Recursos do Desenvolvimento

\begin{tabular}{ll}
\hline Recursos Externos & Recursos Internos \\
\hline Categoria Suporte & Categoria Compromisso com a Aprendizagem \\
Recurso 1: Suporte familiar & Recurso 21: Motivação para a aprendizagem \\
Recurso 2: Comunicação familiar positiva & Recurso 22: Compromisso com a escola \\
Recurso 3: Relações com outros adultos & Recurso 23: Trabalho de casa \\
Recurso 4: Vizinhança atenciosa & Recurso 24: Relação com a escola \\
Recurso 5: Ambiente escolar cuidador & Recurso 25: Satisfação com a leitura \\
Recurso 6: Envolvimento de pais com escola & Categoria Valores Positivos \\
Categoria Empowerment & Recurso 26: Atenção \\
Recurso 7: Comunidade valoriza os adolescentes & Recurso 27: Igualdade e justiça social \\
Recurso 8: Adolescentes como recursos & Recurso 28: Integridade \\
Recurso 9: Serviço aos outros & Recurso 29: Honestidade \\
Recurso 10: Segurança & Recurso 30: Responsabilidade \\
Categoria Limites e Expetativas & Recurso 31: Controlo \\
Recurso 11: Limites na família & Categoria Competências Sociais \\
Recurso 12: Limites na escola & Recurso 32: Planear e tomar decisões \\
Recurso 13: Limites na vizinhança & Recurso 33: Competência interpessoal \\
Recurso 14: Adultos modelo de comportamento & Recurso 34: Competência cultural \\
Recurso 15: Influência positiva dos amigos & Recurso 35: Capacidade de resistência \\
Recurso 16: Elevadas expectativas & Recurso 36: Resolução de conflitos pacífica \\
Categoria Uso Construtivo do tempo & Categoria Identidade Positiva \\
Recurso 17: Atividades criativas & Recurso 37: Poder pessoal \\
Recurso 18: Programas para Adolescentes & Recurso 38: Autoestima \\
Recurso 19: Comunidade religiosa & Recurso 39: Sentido de propósito \\
Recurso 20: Tempo em casa & Recurso 40: Visão positiva do futuro pessoal
\end{tabular}

Nota. Copyright @ 2 2012, Search Institute, Minneapolis, MN; 800-888-7828; www.search-institute.org. All rights reserved. Do not reproduce.

Fundamentado pelas Teorias Sistémicas do Desenvolvimento, que consistem numa metateoria relacional do desenvolvimento humano que propõe uma abordagem integrada na compreensão do desenvolvimento (Ford \& Lerner, 1992; Gottlieb, 1997, Gottlieb, Wahlsten, \& Lickluter, 2006; Lerner \& Steinberg, 2009; Overton, 2006, 1013; Overton \& Müller, 2012), central ao Modelo de Recursos do Desenvolvimento encontra-se a potencial plasticidade ontogenética que reconhece a existência de relações de interdependência recíproca entre os níveis biológico, individual e contextual de organização. O reconhecimento de que a regulação do desenvolvimento envolve relações individuo $\longrightarrow$ contextos mutuamente influentes entre todos os níveis indica, como consequência, o potencial de mudança sistemática, isto é, pela plasticidade e diversidade. Tal pressupõe que o desenvolvimento humano positivo pode ser promovido, através da articulação das características dos indivíduos e das ecologias (Benson et al., 2006), o que constitui de acordo com o Modelo, "a fusão de recursos internos e externos (i.e., ecológicos)" (Benson, 2007, p. 38).

Das formulações teóricas no âmbito das Teorias Sistémicas do Desenvolvimento, emerge também o pressuposto central subjacente ao Modelo de Recursos colocado como "quanto maior a experiência de recursos, melhor" (Benson et al., 2007, p. 38). Este refere-se à abordagem de Construção de Recursos, a qual sugere que quanto maior o número de experiências positivas o adolescente revelar, maior a probabilidade de um desenvolvimento bem-sucedido (Benson et al., 2006, 2011). Benson et al. (2011, p. 204) explicam que "um Recurso do Desenvolvimento constitui um agente ou característica do indivíduo ou das suas ecologias desenvolvimentais (...) que está relacionado com o aumento da probabilidade de resultados positivos", os quais "aumentam 
aditivamente ou exponencialmente à medida que o número de Recursos do Desenvolvimento aumenta".

Dado que a Perceção de Saúde reflete os recursos da pessoa, assim como consiste numa avaliação dinâmica, que consiste num julgamento relativo não apenas à saúde atual, mas o qual reflete a trajetória da saúde, trata-se de um importante resultado a explorar no desenvolvimento durante a adolescência. Uma revisão da literatura revela que poucos estudos exploram a relação entre a Perceção de Saúde e Recursos do Desenvolvimento no contexto da Perspetiva do Desenvolvimento Positivo dos Adolescentes (Soares et al., 2018b). Deste modo, torna-se relevante compreender como cada uma destas importantes áreas se relaciona dadas as implicações para a promoção de um desenvolvimento positivo na adolescência.

De notar ainda que, a maioria da investigação sobre a Perceção de Saúde foi realizada com adultos, salientando-se que compreender Perceções de Saúde em subpopulações específicas, nomeadamente a adolescência, é útil no planeamento de intervenções que visam aumentar o conhecimento em saúde e o envolvimento em comportamentos de saúde (Benyamini, 2011a). Para além da idade, intervenções que visam melhorar a saúde geral podem ser mais eficazes se integrarem questões de género e ações específicas neste domínio (Benyamini, 2011a; Benyamini, Leventhal, \& Leventhal, 2000; Craig et al., 2018; Jerdén et al., 2011; Potrebny, 2019; Sokol et al. 2017; Vingilis et al., 2002)

O objetivo do presente estudo consiste em explorar a relação entre a Perceção de Saúde e a experiência de Recursos do Desenvolvimento, analisando especificamente quais os Recursos Internos e Recursos Externos compreendidos pelo Modelo constituem preditores de Saúde na adolescência.

\section{Método}

\section{Participantes}

Foi avaliada uma amostra de conveniência constituída por 503 estudantes que se encontravam a frequentar o sistema de ensino entre o $9^{\circ}$ e o $12^{\circ}$ ano de escolaridade de uma escola pública da região Norte de Portugal, com idades compreendidas entre os 13 e 19 anos de idade ( $M$ $=16 ; D P=1,2)$, maioritariamente do género feminino $(63 \%)$.

\section{Material}

\section{Perceção de Saúde}

Avaliada com o item de Perceção Geral de Saúde constituinte do questionário SF-36 (primeiro item do questionário), desenvolvido por Ware, Snow, Kosinski e Gandek (1993) e validado para Portugal por Ferreira e Santana, (2003), o qual questiona: "Em geral, como dirias que a tua saúde é?", com opções de resposta numa escala do tipo Likert com cinco posições, que varia entre " $1=$ ótima" a " $5=$ fraca". Pontuações inferiores indicam melhor perceção de saúde. Existem vários motivos para considerar a utilização de um único item de perceção geral da saúde: a principal, em consonância com a investigação realizada por Benyamini (2008, 2011a, 2011b), sugere-se a sua utilidade na medida em que perceções subjetivas das pessoas sobre sua saúde captam fatores físicos, psicológicos e sociais, em consonância com a visão holística da saúde, além de fornecer resumos abrangentes do conjunto de fatores que as pessoas consideram como parte de seu próprio conceito de saúde; a avaliação global da saúde é uma medida genericamente aceite em epidemiologia (Benyamini, 2008; 2011a; 2011b; Idler \& Benyamini, 1997). As estatísticas europeias e mundiais utilizam desde o final do século passado este indicador (Eurostat, 2020). É um indicador integrado em questionários de avaliação da saúde em doenças especificas como, por 
exemplo, no cancro - QLQ-C30 (Aaronson et al., 1993), na esclerose múltipla - MSQOL 54 (Vickrey et al., 1995), na epilepsia - ESI 55 (Vickrey et al., 1992).

\section{Recursos do Desenvolvimento}

Perfis da Vida de Estudante: Atitudes e Comportamentos (A\&B) - Questionário desenvolvido pelo Search Institute - Profile of Student Life: Attitudes and Behaviors ${ }^{\circledR}$ (A\&B) (Benson et al., 1998; Leffert et al., 1998; Search Institute, 2019) e validado para português-europeu por Soares, Pais-Ribeiro e Silva (2018a; 2018c). Dirige-se a adolescentes com idades compreendidas dos 12 aos 18 anos em contexto escolar, ou especificamente, a adolescentes que se encontrem no $2^{\circ}$ e $3^{\circ}$ ciclo do ensino básico, a ensino secundário no sistema de ensino Português (no original, middle school-high school, no sistema de ensino dos Estados Unidos da América) (Leffert et al., 1998).

A avaliação da experiência dos 40 Recursos individuais compreende 92 itens, que consistem, a sua maioria, afirmações relativamente às quais são apresentadas opções de resposta numa escala tipo Likert de cinco posições que variam entre, por exemplo, 1 = concordo muito" e $5=$ discordo muito, 1 = nada importante e $5=$ extremamente importante, $1=$ não sou nada assim e $5=$ sou muito assim.

Existem duas abordagens para descrever a experiência de Recursos: 1) Os dados podem ser analisados assumindo-se que se trata de uma escala do tipo Likert, sendo que o valor obtido em cada um dos Recursos é interpretado da seguinte forma: quanto mais elevada a pontuação, maior a experiência do Recurso; 2) Por sua vez, para fins de comunicação, a pontuação de cada Recurso pode ser utilizada como variável dicotómica, isto é, o adolescente tem ou não tem o Recurso. Desta pontuação de cada um dos 40 Recursos resulta um Índice em que a pontuação varia entre $0-40$ Recursos, o qual descreve o número médio de Recursos que cada adolescente possui. Esta abordagem proporciona ainda a análise do conceito de Níveis de Recursos, o qual reflete o continuum mais amplo de desenvolvimento saudável e define quatro Níveis de Recursos com base no Índice de Recursos que o adolescente experimenta, representando um continuum de desenvolvimento de "At-risk" a "Optimal", assim como o axioma da Construção de Recursos "the more assets, the better": Nível 1 - At-risk Development: 0 a 10 Recursos; Nível 2 - Vulnerable Development: 11 a 20 Recursos; Nível 3 - Adequate Development: 21 a 30 Recursos; e Nível 4 Optimal Development: 31 a 40 Recursos (Benson et al., 1998; Leffert et al., 1998).

$A$ versão portuguesa do questionário $A \& B$ possui características de validade similares às da versão original (Soares et al., 2018c). Vinte e um dos 40 Recursos são medidos por pelo menos 3 itens, a maioria apresenta valores de consistência interna considerados aceitáveis, variando de 0,60 a 0,80 (Cronbach, 1951). Treze Recursos são medidos utilizando-se itens individuais, pelo que tal avaliação não se aplica. Avaliação da consistência interna de Categorias: Suporte $\alpha=0,81$; Empowerment $\alpha=0,76$; Limites e Expectativas $\alpha=0,65$; Uso construtivo do tempo $\alpha=0,39$ (categoria multidimensional); Compromisso com a Aprendizagem $\alpha=0,67$; Valores Positivos $\alpha=$ 0,78; Competências Sociais $\alpha=0,72$; Identidade Positiva $\alpha=0,83$. Análise de Dimensões: Recursos Externos $\alpha=0,84$; Recursos Internos $\alpha=0,84$.

\section{Procedimento}

A aprovação para a realização desta pesquisa foi concedida pela Direção Geral de Educação. Este estudo foi realizado de acordo com os regulamentos relativos à ética profissional, conforme declarado pela Ordem dos Psicólogos Portugueses (2016). A Comissão Nacional de Proteção de Dados confirmou o anonimato dos participantes na recolha de dados, dando o seu parecer favorável. Foram solicitadas autorizações para a recolha de dados ao Conselho Geral e Diretor do Agrupamento de Escolas, e Professores. Pais/responsáveis legais de todos os alunos que participaram do estudo assinaram o termo de consentimento livre e informado para a recolha 
de dados, uso e publicação dos resultados. A recolha de dados foi realizada em sala de aula com instruções padronizadas. Os alunos a quem os pais/responsáveis legais deram consentimento para a participação, foram convidados a responder ao questionário e colocá-lo num envelope. Foi assegurado o anonimato e a confidencialidade dos dados e a sua utilização exclusiva para fins de investigação.

\section{Resultados}

\section{Perceção de Saúde}

A Tabela 2 apresenta os resultados médios (e desvios-padrão entre parênteses) de Perceção de Saúde por género, idade, grau de escolaridade e amostra total. Os dados indicam que os adolescentes apresentam um resultado médio de Perceção de Saúde de 2,25 ( $D P=1,02)$. Considerando-se o género, observam-se diferenças estatisticamente significativas nos resultados médios, os quais indicam que os rapazes $(M=2,02 ; D P=0,92)$ revelam uma melhor Perceção de Saúde comparativamente às raparigas $(M=2,39 ; D P=1,04), t(495)=3,96, p<0,0001$. Verificouse que não existe uma correlação estatisticamente significativa entre a idade e a Perceção de Saúde, $r(501)=0,07, p=0,13$. Os resultados sugerem também a não existência de diferenças estatisticamente significativas nos resultados médios de Perceção de Saúde entre graus de escolaridade, $F(3,496)=1,57, p=0,19$.

Os resultados deste estudo indicam que a maior percentagem de adolescentes (88\% destes) afirma que a sua Perceção de Saúde se encontra entre Boa, Muito Boa e Ótima: $28,7 \%(n=144)$ revela a sua Perceção de Saúde como Ótima; 29,8\% $(n=150)$ revela Muito Boa; $29,4 \%(n=148)$ revela Boa. Os demais, 10,9\% $(n=55)$ revela a sua Perceção de Saúde Razoável e 0,8\% $(n=4)$ revela Fraca.

Tabela 2

Resultados Médios (e Desvios-Padrão entre Parênteses) de Perceção do Estado de Saúde por Género, Idade, Grau de Escolaridade e Amostra Total

\section{Perceção de Saúde}

\begin{tabular}{|c|c|c|c|c|c|c|}
\hline \multicolumn{2}{|l|}{ Género } & \multicolumn{4}{|c|}{ Grau de Escolaridade } & \multirow{2}{*}{$\begin{array}{l}\begin{array}{l}\text { Amostra } \\
\text { Total }\end{array} \\
(N=500) \\
M(D P)\end{array}$} \\
\hline $\begin{array}{l}\text { Masculino } \\
(n=184) \\
M(D P)\end{array}$ & $\begin{array}{l}\text { Feminino } \\
(n=313) \\
M(D P)\end{array}$ & $\begin{array}{l}9^{\circ} \text { Ano } \\
(n=108) \\
M(D P)\end{array}$ & $\begin{array}{l}10^{\circ} \text { Ano } \\
(n=176) \\
M(D P)\end{array}$ & $\begin{array}{l}11^{\circ} \text { Ano } \\
(n=139) \\
M(D P)\end{array}$ & $\begin{array}{l}12^{\circ} \text { Ano } \\
(n=77) \\
M(D P)\end{array}$ & \\
\hline $2,02(0,92)$ & $2,39(1,04)$ & $\begin{array}{l}2,09 \\
(0,93)\end{array}$ & $2,30(1,04)$ & $\begin{array}{l}2,24 \\
(0,99)\end{array}$ & $\begin{array}{l}2,40 \\
(1,10)\end{array}$ & $\begin{array}{l}2,25 \\
(1,02)\end{array}$ \\
\hline
\end{tabular}

\section{Efeito da Experiência Cumulativa de Recursos do Desenvolvimento na Perceção de Saúde}

A Tabela 3 apresenta os resultados médios (e desvios-padrão entre parênteses) de Perceção de Saúde na amostra total de adolescentes e por Nivel de Recursos que os adolescentes revelam possuir - isto é, os quatro Níveis de Recursos definidos como 0-10, 11-20, 21-30 e 31-40 Recursos. Para analisar o impacto cumulativo da experiência de Recursos de Desenvolvimento na Perceção de Saúde, foi realizada a análise de variância (one-way ANOVA, Post-Hoc Bonferroni). Os resultados indicam a existência de diferenças estatisticamente significativas em função do número de Recursos experimentado pelos adolescentes nos diferentes grupos, $F(3,463)=15,78, p<$ 
0,0001 (o teste Post-Hoc Bonferroni indica que todos os grupos diferem significativamente entre si), observando-se que os adolescentes com Níveis mais elevados de Recursos, isto é, que experimentam um maior número de Recursos, tendem a revelar uma melhor Perceção de Saúde.

Tabela 3

Médias (Desvios-Padrão entre Parênteses) de Perceção de Saúde por Nível de Recursos

\begin{tabular}{|c|c|c|c|c|c|}
\hline \multirow{5}{*}{$\begin{array}{l}\text { Amostra Total } \\
(N=503) \\
M(D P)\end{array}$} & \multicolumn{5}{|c|}{ Perceção de Saúde por Nível de Recursos } \\
\hline & Nível 1 & Nível 2 & Nível 3 & Nível 4 & \\
\hline & $0-10$ & $11-$ & $21-$ & $31-$ & \\
\hline & $(n=28)$ & $(n=238)$ & $(n=185)$ & $(n=16)$ & $F$ \\
\hline & $M(D P)$ & $M(D P)$ & $M(D P)$ & $M(D P)$ & \\
\hline $2,25(1,02)$ & $3,04(0,84)$ & $2,42(1,01)$ & $1,98(0,97)$ & $1,56(0,63)$ & $15,78^{*}$ \\
\hline
\end{tabular}

Nota. Resultados inferiores indicam melhor Perceção de Saúde; * $p<0,01$

\section{Recursos do Desenvolvimento Preditores de Saúde}

Para determinar que Recursos do Desenvolvimento e que variáveis demográficas prediziam resultados de Perceção de Saúde, utilizou-se a análise de regressão múltipla stepwise (Tabela 4). Foram incluídos dois conjuntos de variáveis preditoras. O primeiro incluiu como variáveis preditoras as medidas demográficas (género, idade e grau de escolaridade do adolescente) e o segundo, em adição, os 40 Recursos do Desenvolvimento. Os resultados sugerem o género (masculino) como preditor independente e significativo da Perceção de Saúde nos adolescentes, representando este fator 3\% da variância nos resultados. A adição das variáveis de Recursos revelou que o Recurso Interno de Autoestima deu a maior contribuição para a predição de Perceção de Saúde, assim como os Recursos de Visão Positiva do Futuro Pessoal, Planear e Tomar Decisões, experiência de Segurança, participação em Programas para Adolescentes e experiência de Adolescentes como Recursos também produziram previsibilidade significativa. O modelo final explicou $23 \%$ da variância nos resultados de Perceção de Saúde dos adolescentes: $R 2=0,24$; $R 2($ Adj. $)=0,23, F(7,462)=20,5, p<0,0001$.

Tabela 4

Preditores de Perceção de Saúde (Análise de Regressão Stepwise)

Nota. ${ }^{*} p<0,05,{ }^{*} p<0,01$

\begin{tabular}{llllll}
\hline & & \multicolumn{3}{l}{ Perceção de Saúde } & \\
\cline { 2 - 6 } & Preditor & \multicolumn{1}{l}{$t$} & $\Delta R^{2}$ & $R^{2}$ (Adj) \\
\hline 1 & Género & 0,03 & 0,71 & 0,02 & 0,02 \\
2 & Autoestima & 0,18 & $3,59 * *$ & 0,13 & 0,15 \\
3 & Visão positiva do futuro pessoal & 0,13 & $2,74^{* *}$ & 0,03 & 0,18 \\
4 & Planear e tomar decisões & 0,12 & $2,80^{* *}$ & 0,02 & 0,20 \\
5 & Segurança & 0,13 & $2,94^{* *}$ & 0,01 & 0,21 \\
6 & Programas para adolescentes & 0,10 & $2,44^{* *}$ & 0,01 & 0,22 \\
7 & Adolescentes como recursos & 0,11 & $2,28^{*}$ & 0,01 & 0,23 \\
\hline
\end{tabular}




\section{Discussão}

Os resultados deste estudo indicam que a maior percentagem de adolescentes $(88 \%$ dos adolescentes) afirma que a sua Perceção de Saúde se encontra no intervalo positivo, isto é, entre Boa, Muito Boa e Ótima. Observa-se que a média de Perceção de Saúde dos adolescentes se encontra no intervalo correspondente a Muito Boa. No seguimento das previsões deste estudo, os resultados indicam que uma melhor Perceção de Saúde está associada a uma maior experiência de Recursos de Desenvolvimento. Estes sugerem que quanto maior o número de Recursos de Desenvolvimento o adolescente revela experimentar, maior a probabilidade de também revelar melhor Perceção de Saúde, pelo que estes dados suportam assim o pressuposto da natureza aditiva dos Recursos em relação ao seu impacto sobre resultados positivos no desenvolvimento em adolescentes, estendendo o conhecimento especificamente a resultados de Perceção de Saúde. Por sua vez, estes resultados vão ao encontro da sugestão de Benyamini (2011a) de que resultados de Perceção de Saúde refletem os recursos da pessoa.

Considerando-se variáveis demográficas os dados do presente estudo sugerem que apenas a variável género contribui para a variância nos resultados de Perceção de Saúde. Estudos anteriores com indivíduos adultos sugerem a existência de diferenças de género na Perceção de Saúde, tratando-se de um preditor de saúde mais forte entre homens comparativamente a mulheres (Benyamini et al., 2000). Em adolescentes, a investigação sobre diferenças de género e idade na Perceção de Saúde conduziu a resultados não consensuais. Por um lado, em linha com os resultados do presente estudo, a maioria dos estudos indica que, os rapazes classificam a sua saúde em níveis mais elevados comparativamente às raparigas (Benyamini, 2009; Benyamini et al., 2000; Craig et al., 2018; Currie et al., 2012; Jardén et al., 2011; Vingilis et al., 2002). embora alguns estudos indiquem que o efeito causal diminua com a inclusão de outros fatores pessoais e contextuais (Breidablik, Meland, \& Lydersen, 2009; Vingilis et al., 2002). Por sua vez, não se observaram diferenças significativas relativamente à idade e grau de escolaridade, assim como a variável idade não contribui para a variância nos resultados de Perceção de Saúde. Estes resultados podem teoricamente sugerir que este estudo se centrou num grupo homogéneo de adolescentes que foram expostos a um contexto similar em experiências.

Os resultados de estudos anteriores são inconsistentes na trajetória de Perceção de Saúde considerando-se género e idade ao longo da adolescência. Jardén et al. (2011) observaram que durante a adolescência, as raparigas relataram a sua saúde em níveis mais baixos do que os rapazes e esta diferença de género aumentou ao longo dos anos. Por sua vez, no estudo desenvolvido por Potrebny et al. (2019), uma menor proporção de raparigas comparativamente aos rapazes classificou sua saúde como excelente, verificando-se, que, com o tempo, no entanto, a proporção de rapazes que avaliaram a sua saúde como excelente diminuiu, enquanto as avaliações das raparigas melhoraram. Também neste sentido, no estudo de Sokol et al. (2017) desenvolvido com o objetivo de observar as trajetórias de Perceção de Saúde dos 13 aos 31 anos, os resultados indicaram que os homens tiveram melhor Perceção de Saúde inicial em comparação com as mulheres, mas, no entanto, estes também experimentaram maiores declínios na Perceção de Saúde ao longo do tempo. Os autores sugeriram que tal pode ser devido ao surgimento de ideais de masculinidade hegemónicos, associados em estudos anteriores a comportamentos de risco de saúde (Heath et al., 2017; Kreager \& Staff, 2009; Wells et al., 2014). Teoricamente, esta compreensão de género remete a uma visão construcionista de género, em que exigências com base em género criam diferentes formas de feminilidades e masculinidades (Connell, 2014).

Breidablik et al. (2009) observaram que ao longo de 4 anos as raparigas indicaram uma deterioração da Perceção de Saúde com mais frequência do que os rapazes, contudo, esta diferença entre os sexos, assim como as diferenças de grupo de idade, tornaram-se não significativas em modelos multivariados, levando os autores a concluir que Perceção de Saúde se trata de um constructo relativamente estável durante a adolescência e que esta se deteriora consistentemente 
com a falta de bem-estar geral, incapacidade, frequência de serviços de cuidados de saúde, e fatores de estilo de vida comprometedores da saúde.

Diferenças biológicas e sociais entre mulheres e homens, bem como a interação entre estas, levam a diferenças de género nas perceções de saúde e doença. Estes fatores também podem interagir, de modo que diferentes sintomas, ou doenças, experimentados num contexto social diferente, que gera expectativas baseadas em género, podem levar a diferenças maiores do que as observadas pelos fatores biológicos ou sociais separadamente (Benyamini, 2011a).

Deste modo, os resultados deste estudo sugerem que intervenções destinadas a melhorar a saúde geral dos adolescentes podem ser mais eficazes se considerarem ações específicas a género. Por sua vez, salienta-se que os efeitos de género e idade na Perceção de Saúde, podem depender do contexto sociocultural específico (Benyamini, 2011a; Potrebny et al., 2019) como tal, sugerese a necessidade de realização de mais investigação na análise desta questão. Do ponto de vista da saúde pública, é também importante reconhecer as possíveis influências de nível macro na saúde e procurar políticas públicas promotoras de saúde. Como explica Benyamini (2011a), parece impossível compreender Perceções de Saúde sem considerar o contexto cultural nos quais são formuladas.

Por sua vez, os dados sugerem o impacto de um subconjunto de Recursos do Desenvolvimento na predição de Saúde dos adolescentes, indicando que ambos os Recursos Internos e Externos contribuíram para a variância nos resultados, constituindo os Recursos Internos preditores mais fortes. O Recurso Interno de Autoestima, na categoria de Identidade Positiva, constitui o preditor mais forte de Perceção de Saúde. Estes resultados são congruentes com a investigação que sugere o componente mais importante da perceção de saúde/doença é a identidade (Benyamini, 2011a; Rees, Fry, Cull, \& Sutton, 2004). Adicionalmente, o Recurso de Visão Positiva do Futuro Pessoal, também na categoria de Identidade Positiva, o qual indica em que medida o adolescente é otimista relativamente ao seu futuro pessoal, também contribuiu para a predição de Saúde. Benyamini et al. (2011) explicam que é esperado que a perceção de saúde esteja relacionada com a visão do mundo e com expetativas generalizadas para o futuro, uma vez que esta envolve perceções e expetativas no presente e no futuro, específicas para a condição de saúde. Relacionado também com recursos internos, os resultados destacam a importância de Competências Sociais envolvendo a capacidade pessoal de Planear e Tomar Decisões, a qual demostra a capacidade do adolescente de planear antecipadamente e fazer escolhas, na predição de Saúde. Em linha com estes resultados, Benyamini et al. (2011) sugerem que, manter um sentimento de mestria e controlo, mesmo apesar de uma saúde muito fraca, pode constituir um indicador ou um fator causal de sobrevivência.

Do mesmo modo, os Recursos Externos, os quais proporcionam a condições do contexto ou ambiente de suporte para otimizar a saúde também produziram previsibilidade significativa. Os resultados destacam a importância da experiência de Segurança, isto é, de os adolescentes se sentirem seguros em casa, na escola, na vizinhança e na comunidade. Adolescentes que expressam sentimento de segurança têm uma maior probabilidade de se sentirem saudáveis do que aqueles que percecionam a sua segurança ameaçada (Benson, 2006; Simonsen et al., 2017). A experiência de participação em Programas para Adolescentes, relativa à categoria de Uso Construtivo do Tempo, a qual traduz a importância da participação em desportos, clubes ou organizações na escola e/ou na comunidade também produziu previsibilidade significativa. Estes resultados vão ao encontro de estudos anteriores que sugerem a atividade física como um importante fator que afeta mudanças na perceção de saúde e prediz menor mortalidade (Benyamini et al., 2011). Por sua vez, em linha com estudos anteriores (Simonsen et al., 2017) os resultados destacam também a experiência de Adolescentes como Recursos, a qual foca a capacidade da comunidade proporcionar oportunidades que permitam ao adolescente contribuir significativamente para a sociedade, assim como indica em que medida a comunidade é favorável ao desempenho de um papel ativo por parte dos adolescentes, consistindo também um importante preditor de saúde. 
Os resultados deste estudo devem ser interpretados tendo em consideração as limitações seguidamente expostas. Dado o desenho transversal deste estudo, não podem ser inferidas relações causais entre os resultados de Perceção de Saúde e a experiência de Recursos do Desenvolvimento. Torna-se necessário o desenvolvimento de estudos longitudinais para determinar se resultados de Perceção de Saúde constituem uma consequência ou um determinante da experiência de Recursos do Desenvolvimento.

\section{Conclusão}

Perceções de Saúde, como Benyamini (2011a, p. 305) explica, organizam o nosso conhecimento e experiência relacionados com a saúde em estruturas significativas que utilizamos para interpretar novas experiências e determinar como lidar com estas, consistindo deste modo, o componente principal e central do processo dinâmico de autorregulação da saúde.

A Perceção de Saúde é, como notamos, o produto da interação entre características pessoais e ações de agentes sociais, como famílias, sistema educacional e condições contextuais. Sugere-se a utilidade do Modelo de Recursos do Desenvolvimento na compreensão desta dinâmica de processo de autorregulação de efeitos internos (self-system) e externos (sistema sociocultural). De notar também que, Perceções de Saúde refletem os recursos da pessoa, os quais refletem ou até mesmo afetam a capacidade de lidar com ameaças à saúde (Benyamini, 2011b). Deste modo, o Modelo de Recursos do Desenvolvimento proporciona assim uma abordagem holística na compreensão de desenvolvimento positivo dos adolescentes, apresentando um quadro conceptual e metodológico com uma conceção do adolescente baseada nas suas forças, com atenção aos fatores que favorecem o desenvolvimento ótimo dos adolescentes, isto é, com foco em Recursos que podem ser explorados quer ao nível individual, quer dos contextos desenvolvimentais (tais como familiar, escolar, comunitário) de modo a propiciar aos adolescentes um desenvolvimento positivo (Benson, 2006; Benson et al., 2006; Benson \& Scales, 2011; Benson et al., 2011). De notar que, estes Recursos foram selecionados por constituírem experiências, relações, habilidades positivas que podem ser promovidas por aqueles próximos dos jovens, nomeadamente pais, professores, outros adultos significativos, não dependendo de influências de nível macro na saúde. Os adultos estão numa posição chave para criar ambientes onde os adolescentes experimentem estas experiências positivas. Importante sublinhar que, estes Recursos da pessoa, externos e internos, constituem assim fatores que podem levar a diferentes trajetórias de saúde futura (Benyamini, 2011b).

Sugere-se a aplicabilidade e utilidade do Modelo de Recursos do Desenvolvimento para a compreensão da Perceção de Saúde em adolescentes, assim como da estratégia de Construção de Recursos, tanto em procurar construir todos os 40 Recursos na vida dos adolescentes, como em promover Recursos específicos com o propósito de favorecer o alcançar de estado e processo de desenvolvimento ótimo na adolescência.

\section{Referências}

Aaronson, N, Ahmedzai, S., Bergman, B., Bullinger, Cull, A, Duez, N. ... Takeda,F (1993). The European Organization for Research and Treatment of Cancer QLQ-C30: A quality of life instrument for use in international clinical trials in oncology. Journal of the National Cancer Institute, 85(5), 365-375. doi: 10.1093/jnci/85.5.365

Benson, P. L. (2006). All kids are our kids: What communities must do to raise caring and responsible children and adolescents ( ${ }^{\text {nd }}$ ed.). San Francisco, CA: Jossey-Bass.

Benson, P. L. (2007). Developmental Assets: An overview of theory, research, and practice. In R. K. Silbereisen \& R. M. Lerner (Eds.), Approaches to Positive Youth Development (pp. 3358). Thousand Oaks, CA: Sage Publications Ltd. doi: 10.4135/9781446213803.n2 
Benson, P. L., Leffert, N., Scales, P. C., \& Blyth, D. A. (1998). Beyond the "village" rhetoric: Creating healthy communities for children and adolescents. Applied Developmental Science, 2, 138-59. doi: 10.1207/s1532480xads0203_3

Benson, P. L., \& Scales, P. C. (2009). Positive youth development and the prevention of youth aggression and violence. International Journal of Developmental Science, 3, 218-234. doi: 10.3233/DEV-2009-3302

Benson, P. L., Scales, P. C., Hamilton, S. F., \& Sesma, Jr., A. (2006). Positive youth development: Theory, research and applications. In W. Damon, \& R. M. Lerner (Eds.), Handbook of child psychology. Theoretical models of human development (6 ${ }^{\text {th }}$ ed.), (pp. 894-941). New York, NY: John Wiley. doi: 10.1002/9780470147658.chpsy0116.

Benyamini, Y. (2008). Self-ratings of health and longevity: A health psychologist's viewpoint on epidemiological findings. European Health Psychologist, 10, 10-12.

Benyamini, Y. (2009). Stress and Coping with Women's Health Issues: A Review from a SelfRegulation Perspective. European Psychologist, 14, 63-71. doi: 10.1027/10169040.14.1.63

Benyamini, Y. (2011a). Perceptions of health and illness. In H.S. Friedman (Ed.), The Oxford handbook of health psychology (pp. 285-318). New York, NY: Oxford University Press.

Benyamini, Y. (2011b). Why does self-rated health predict mortality? An update on current knowledge and a research agenda for psychologists. Psychology \& Health, 26 (11), 14071413. doi: 10.1080/08870446.2011.621703

Benyamini, Y., Blumstein, T., Murad, H., \& Lerner-Geva, L. (2011). Changes over time from baseline poor self-rated health: For whom does poor self-rated health NOT predict mortality? Psychology and Health, 26, 1446-1462. doi: 10.1080/08870446.2011.559231

Benyamini, Y., \& Idler, E.L. (1999). Community studies reporting association between self-rated health and mortality: Additional studies, 1995-1998. Research on Aging, 21, 392-401. doi: $10.1177 / 0164027599213002$

Benyamini, Y., Idler, E. L., Leventhal, H., \& Leventhal, E. A. (2000). Positive affect and function as influences on self-assessments of health: Expanding our view beyond illness and disability. The Journals of Gerontology Series B: Psychological Sciences and Social Sciences, 55, 107-116. doi: 10.1093/geronb/55.2.P107

Benyamini, Y., Leventhal, E. A., \& Leventhal, H. (2000). Gender differences in processing information for making self-assessments of health. Psychosomatic Medicine, 62, 354-364. doi: 10.1097/00006842-200005000-00009

Bishop, F., \& Yardley, L. (2010). The development and initial validation of a new measure of lay definitions of health: The wellness beliefs scale. Psychology and Health, 25, 271-287.

Breidablik, H. J., Meland, E., \& Lydersen, S. (2009). Self-rated health during adolescence: stability and predictors of change (Young-HUNT study, Norway). The European Journal of Public Health, 19(1), 73-78. doi: 10.1093/eurpub/ckn111

Connell, R. W. (2014). Gender and power: Society, the person and sexual politics. Oxford: John Wiley \& Sons.

Craig, B. A., Morton, D. P., Morey, P. J., Kent, L. M., Gane, A. B., Butler, T. L., ... \& Price, K. R. (2018). The association between self-rated health and social environments, health behaviors and health outcomes: a structural equation analysis. BMC Public Health, 18, 440. doi: 10.1186/s12889-018-5323-y

Cronbach, L. J. (1951). Coefficient alpha and the internal structure of tests. Psychometrika, 16, 297-334. doi: 10.1007/BF02310555

Currie, C., Zanotti, C., Morgan, A., Currie, D., De Looze, M., Roberts, C., ... \& Barnekow, V. (2012). Social determinants of health and well-being among young people. Health Behaviour in School-aged Children (HBSC) study: international report from the 
2009/2010 survey. Copenhagen: WHO Regional Office for Europe, Health Policy for Children and Adolescents.

Doiron, D., Fiebig, D. G., Johar, M., \& Suziedelyte, A. (2015). Does self-assessed health measure health? Applied Economics, 47, 180-194. doi: 10.1080/00036846.2014.967382

Eurostat (2020). Eurostat statistics explained. Retrieved from:https://ec.europa.eu/eurostat/statistics-explained/index.php?title=Glossary:Selfperceived_health

Ferreira, P., \& Santana, P. (2003). Percepção do estado de saúde e de qualidade de vida da população activa: Contributo para a definição de normas portuguesas. Revista Portuguesa de Saúde Pública, 21, 15-30.

Ford, D. H., \& Lerner, R. M. (1992). Developmental systems theory: An integrative approach. Newbury Park, CA: Sage Publications.

Gottlieb, G. (1997). Synthesizing nature-nurture: Prenatal roots of instinctive behavior. Mahwah, NJ: Lawrence Erlbaum.

Gottlieb, G., Wahlsten, D. \& Lickluter, R. (2006). The significance of biology for human development: A developmental psychobiological systems perspective. In R. M. Lerner (Ed.) Theoretical models of human development: Handbook of Child Psychology (6 ${ }^{\text {th }}$ ed.) (vol.1, pp. 210-257). Hoboken, NJ: Wiley.

Heath, P. J., Brenner, R. E., Vogel, D. L., Lannin, D. G., \& Strass, H. A. (2017). Masculinity and barriers to seeking counseling: The buffering role of self-compassion. Journal of Counseling Psychology, 64 (1), 94-103. doi: 10.1037/cou0000185

Idler, E. L., \& Benyamini, Y. (1997). Self-rated health and mortality: a review of twenty-seven community studies. Journal of health and social behavior, 38, 21-37. doi: $10.2307 / 2955359$

Jerdén, L., Burell, G., Stenlund, H., Weinehall, L., \& Bergström, E. (2011). Gender differences and predictors of self-rated health development among Swedish adolescents. Journal of Adolescent Health, 48, 143-150. doi: 10.1016/j.jadohealth.2010.06.005

Joffer, J., Jerdén, L., Öhman, A., \& Flacking, R. (2016). Exploring self-rated health among adolescents: a think-aloud study. BMC Public Health, 16, 156. doi: 10.1186/s12889-016$2837-\mathrm{Z}$

Jylhä, M. (2009). What is self-rated health and why does it predict mortality? Towards a unified conceptual model. Social, Science Medicine, 69, 307-316. doi: 10.1016/j.socscimed.2009.05.013.

Kreager, D.A., \& Staff, J. (2009). The sexual double standard and adolescent peer acceptance. Social Psychology Quarterly, 72(2), 143-164. doi: 10.1177/019027250907200205

Lerner, R.M., \& Steinberg, L. (2009). The scientific study of adolescent development. In R. M.., Lerner \& L. Steinberg (Eds.), Handbook of adolescent psychology ( $3^{\text {rd }}$ ed.), (Vol. 1, pp. 314). New Jersey, NJ: John Wiley \& Sons, Inc. doi: 10.1002/9780470479193.adlpsy001002

Ordem dos Psicólogos Portugueses (2016). Código Deontológico da Ordem dos Psicólogos Portugueses. $\quad$ Retrieved from https://www.ordemdospsicologos.pt/ficheiros/documentos/web_cod_deontologico_pt_rev isao_2016.pdf

Overton, W.F. (2006). Developmental psychology: Philosophy, concepts, and methodology. In W. Damon \& R. M. Lerner (Eds.), Theoretical Models of Human Development. Handbook of child psychology ( $5^{\text {th }}$ ed.), (Vol. 1, pp.107-188). Hoboken, NJ: Wiley.

Overton, W.F. (2013). A new paradigm for developmental science: Relationism and relationaldevelopmental systems. Applied Developmental Science, 17, 94-107. doi: 10.1080/10888691.2013.778717 
Overton, W.F., \& Müller, U. (2012). Metatheories, Theories, and Concepts in the Study of Development. In Irving B. Weiner (Ed.), Handbook of Psychology ( $2^{\text {nd }}$ ed), (Vol. 6, pp. 1958). Hoboken, NJ: John Wiley \& Sons, Inc. doi: 10.1002/9781118133880.hop206002

Pais-Ribeiro, J.L. (2005). O importante é a Saúde: Estudo de adaptação de uma técnica da perceção do Estado de Saúde. Lisboa: Fundação Merck Sharp \& Dohme.

Pinquart, M. (2001). Correlates of subjective health in older adults: A meta-analysis. Psychology and Aging, 16, 414-426. doi: 10.1037/0882-7974.16.3.414

Potrebny, T., Torsheim, T., Due, P., Välimaa, R., Suominen, S., \& Eriksson, C. (2019). Trends in excellent self-rated health among adolescents: A comparative Nordic study. Nordisk välfärdsforskning| Nordic Welfare Research, 4(02), 67-76. doi: 10.18261/issn.2464-41612019-02-04

Rees, G., Fry, A., Cull, A., \& Sutton, S. (2004). Illness perceptions and distress in women at increased risk of breast cancer. Psychology \& Health, 19, 749-765. doi: 10.1080/08870440412331279764

Scales, P.C., Benson, P. L., Leffert, N., \& Blyth, D.A. (2000). Contribution of developmental assets to the prediction of thriving among adolescents. Applied Developmental Science, 4, 27-46. doi: 10.1207/S1532480XADS0401_3

Scales, P.C., Benson, P.L., Roehlkepartain, E.C., Sesma, A., \& van Dulmen, M. (2006). The role of developmental assets in predicting academic achievement: A longitudinal study. Journal of Adolescence, 29, 691-708. doi: 10.1016/j.adolescence.2005.09.001

Search Institute (2019). User guide for the Attitudes \& Behaviors survey. Retrieved from http://www.search-institute.org/sites/default/files/a/A\&B-Survey-User-Guide.pdf

Simonsen, N., Ylönen, A., Suominen, S., Roos, E., Välimaa, R., Tynjälä, J., \& Kannas, L. (2017). Associations between empowerment-enabling environments and self-rated health among adolescents. European Journal of Public Health, 27(3), ckx187.728. doi: 10.1093/eurpub/ckx187.728

Soares, A.S., Pais-Ribeiro, J.L., \& Silva, I. (2018a). Recursos do desenvolvimento na adolescência: Revisão integrativa. Ciências Psicológicas, 12, 45-57. doi: 10.22235/cp. v12i1.1594

Soares, A.S., Pais-Ribeiro, J.L., \& Silva, I. (2018b). Adaptação portuguesa do questionário Profile of Student Life: Attitudes and Behaviors $(A \& B)$. Manuscript submitted for publication.

Sokol, R., Ennett, S., Gottfredson, N., \& Halpern, C. (2017). Variability in self-rated health trajectories from adolescence to young adulthood by demographic factors. Preventive Medicine, 105, 73-76. doi: 10.1016/j.ypmed.2017.08.015.

Vickrey, B.G., Hays, R.D-, Graber, J., Rausch, R., Engel, J. Jr, Brook. R.H. (1992). A healthrelated quality of life instrument for patients evaluated for epilepsy surgery. Medical Care, 30, 299-319. doi: 10.1097/00005650-199204000-00002.

Vickrey, B.G., Hays, R.D., Harooni, R., Myers, L.W., \& Ellison, G.W. (1995). A health-related quality of life measure for multiple sclerosis. Quality of Life Research, 4, 187-206. doi: 10.1007/BF02260859

Vingilis, E. R., Wade, T. J., \& Seeley, J. S. (2002). Predictors of adolescent self-rated health: analysis of the National Population Health Survey. Canadian Journal of Public Health/Revue Canadienne de Santé Publique, 93, 193-197.

Wells, S., Flynn, A., Tremblay, P. F., Dumas, T., Miller, P., \& Graham, K. (2014). Linking masculinity to negative drinking consequences: The mediating roles of heavy episodic drinking and alcohol expectancies. Journal of Studies on Alcohol and Drugs, 75(3), 510519. doi: 10.15288/jsad.2014.75.510

Ware, J. E., Snow, K. K., Kosinski, M., \& Gandek, B. (1993). SF-36® health survey manual and interpretation guide. Boston, MA: New England Medical Center, the Health Institute. 
Participação dos autores: a) Planejamento e concepção do trabalho; b) Coleta de dados; c) Análise e interpretação de dados; d) Redação do manuscrito; e) Revisão crítica do manuscrito.

A.S. contribuiu em a,b,c,d,e; J.L.P-R. em a,b,c,d,e; I.S. em a,b,c,d,e.

Editora científica responsável: Dra. Cecilia Cracco 